\title{
Betel quid/areca nut chewing and deleterious impact on oral health: is socio-cultural fabric the culprit?
}

\begin{abstract}
The habit of betel quid/areca nut chewing dates back to thousands of years, and is considered by the chewers to be of social, cultural and religious importance. Areca nut use has been reported to cause significant deleterious effects on general as well as oral health. Evidence has revealed that there is an increasing prevalence of areca nut/betel nut chewing and oral cancer. This article explores the socio-cultural implications associated with betel quid/areca nut chewing practice and highlights the need for increased oral health service, active oral health prevention and rehabilitative treatment services in a culturally competent manner to control this habit.
\end{abstract}

Keywords: betel quid, areca nut, chewing habit, oral cancer, culture
Volume 5 Issue I - 2016

\author{
Sandeep Anant Lawande \\ Department of Periodontics, Goa Dental College \& Hospital, \\ India
}

Correspondence: Sandeep Anant Lawande, Department of Periodontics, Goa Dental College \& Hospital, H.No. 874/5, Saideep, New Pundalik Nagar, Porvorim, Goa, India, Tel 91 0989022373I,Email drsanlaw@rediffmail.com

Received: August 27, 2016 | Published: September 12, 2016

\section{Introduction}

Globally, it is estimated that approximately 600 million people are involved in betel quid/areca nut chewing on a habitual basis. ${ }^{1}$ Areca nut is obtained from Areca catechu, a tropical palm tree whereas traditional betel quid ('paan' in local parlance) consists of a mixture of areca nut, slaked lime (calcium hydroxide) and different flavouring agents with or without tobacco enclosed in a Piper betel leaf. The preparations of betel quid may also vary according to the local preferences and practices. Interestingly, there is a wide variety of commercially packaged raw areca nut products in the form of gutka, mawa, khaini, and paan masala available for the users, mostly in India and other parts of the world. Not only men are habitual chewers, but also women, adolescents and even children, among certain communities develop a liking towards areca nut., ${ }^{2,3}$ Areca nut use is most predominant in the Indian subcontinent, East and Southeast Asia and the Pacific Islands and may also include Asian immigrant communities in Europe as well as the United States. ${ }^{4}$ The chewing practice, being centuries old, is considered to be socially acceptable with its amalgamation into the daily routine and finding a special cultural status for the religious ceremonies, owing to which, fear of negative social percussions associated with quitting this habit may exist. $^{5}$ Murphy et al., ${ }^{6}$ examined psychosocial and socio-cultural factors affecting areca nut chewers and established a link with social cognitive theory through which it was revealed that areca chewing was a learned behaviour that was influenced by other chewers. ${ }^{6}$ Areca nut is the fourth most psychoactive substance worldwide after nicotine, alcohol and caffeine and has been found to influence both the sympathetic and parasympathetic nervous systems causing both stimulatory and relaxing effects. ${ }^{2,3}$ The reasons for chewing may include feeling of euphoria, feeling of warmth inside the body, oral fixation, rituals associated with preparation and a desire to prevent withdrawal symptoms. ${ }^{6}$ Epidemiologic studies have revealed betel quid/areca nut chewing to be associated with a number of deleterious effects on the general as well as oral health. As far as oral health is concerned, chewing is linked to an array of disorders including chewer's mucosa, periodontal disease, oral leukoplakia, oral submucous fibrosis, and different types of oral and oropharyngeal cancer. ${ }^{3,5}$ According to the World Health Organization and the International Agency for Research on Cancer (IARC), betel quid/areca nut chewing has been classified as Group 1 human carcinogen. ${ }^{3}$ The alkaloid components of areca nut, which produce nitrosamine derivatives, are responsible for potential carcinogenic effects. Evidence has revealed that there is an increasing prevalence of areca nut/betel nut chewing and oral cancer. ${ }^{2-5}$ Swerdlow et al, in a review of the approximately 1.5 million cancer deaths in England and Wales from the years 1973-1985 showed that Indian-born men had over two times, and Indian-born women over five times, greater risks of oral cancer mortality than native English and Welsh individuals. ${ }^{7}$ Curbing the continuing menace of areca use is the need of the hour and for this, significant proactive steps need to be taken on a priority basis. It is important that certain socio-cultural factors deleteriously affecting oral health in general and those related to the areca nut/betel quid chewing practice in particular be identified and recognized. This will facilitate the formulation of oral health promotion policies through well-conceptualized and appropriate preventive (including patient education and oral cancer screening) as well as therapeutic strategies. ${ }^{6-9}$ It is important to understand cultural perceptions of oral health in order to manage transcultural barriers that may arise during the utilization of oral health care practices. For this, cultural competence should be achieved through the knowledge of various cultural backgrounds in a diverse population. The utmost responsibility of the dental professionals lies in the development of clinical expertise and also the ability to elicit, recognize, accept and respect the cultural beliefs of their patients. ${ }^{10,11}$ More stringent forms of legislation against open sale and use of areca nut products may be urgently needed by the affected countries across the globe. In addition to educating consumers about the health risks of areca nut use, dental professionals need to educate areca nut/betel quid vendors about the repercussions of areca consumption and persuade them not to sell these products to minors. The mass media in the form of radio, television, internet, newspapers, health exhibitions and role plays may be of tremendous help in educating and changing the attitude and 
behaviour of the chewers. ${ }^{8}$ Areca nut/betel quid risk reduction as well as cessation programs, with an elaborate consideration of chewers' cultural status need to be developed worldwide, particularly in the predominant areas. Cognitive behavioural therapy (CBT) approaches have been used successfully for smoking cessation and may be adapted for areca chewing cessation with addition of pharmacotherapy, whenever required. ${ }^{6}$ In cases where even the best culturally competent care is practiced, challenges may arise when there is a need to enhance motivation to change health behaviours in patients who are not motivated to change. In those situations, there may be great value in applying a specific motivational intervention, such as 'motivational interviewing', in order to enhance the motivation of patients to change health behaviours in those patients who are resistant or not motivated to change. This intervention is patient-centered and is directly tailored to patients' readiness to change behavior. ${ }^{3,11}$

\section{Funding}

None.

\section{Acknowledgments}

None.

\section{Conflicts of interest}

Author declares that there is no conflict of interest.

\section{References}

1. Nelson BS, Heischober B. Betel nut: a common drug used by naturalized citizens from India, Far East Asia, and the South Pacific Islands. Ann Emerg Med. 1999;34(2):238-243.
2. Gupta PC, Warnakulasuriya S. Global epidemiology of areca nut usage. Addict Biol. 2002;7(1):77-83.

3. Tobacco habits other than smoking: Betel-quid and Areca-nut chewing and some related nitrosamines. IARC monographs on the evaluation of the carcinogenic risks to humans. International Agency for Research on Cancer (IARC): Lyon, France, IARC, Europe 37; 1985. 1-296 p.

4. Priebe $\mathrm{S}$, Niene JA, Dharamsi S, et al. Oral cancer and cultural factors in Asia. Can J Dent Hygiene. 2008;42(6):291-295.

5. Sudha S, Mythili B, Balachandar V. Mixture of betel leaf, areca nut and tobacco chewing is a risk factor for cytogenetic damage in construction workers from south India. Braz J Oral Sci. 2009;8(3):145-148.

6. Murphy KL, Herzog TA. Sociocultural factors that affect chewing behaviour among betel nut chewers and ex-chewers on Guam. Hawaii $J$ Med Public Health. 2015;74(12):406-411.

7. Swerdlow AJ, Marmot MG, Grulich AE, et al. Cancer mortality in Indian and British ethnic immigrants from the Indian subcontinent to England and Wales. Br J Cancer. 1995;72(5):1312-1319.

8. Anand R, Dhingra C, Prasad S, et al. Betel nut chewing and its deleterious effects on oral cavity. J Can Res Ther. 2014;10(3):499-505.

9. Little MA, Pokhrel P, Murphy KL, et al. The reasons for betel-quid chewing scale: assessment of factor structure, reliability and validity. BMC Oral Health. 2014;14:62.

10. Rayman S, Almas K. Transcultural barriers and cultural competence in dental hygiene practice. J Contemp Dent Pract. 2007;8(4):43-51.

11. Garcia RI, Cadoret C, Henshaw M. Multicultural issues in oral health. Dent Clin North Am. 2008;52(2):319. 